\title{
STMN1, a prognostic predictor of esophageal squamous cell carcinoma, is a marker of the activation of the PI3K pathway
}

\author{
WENPENG JIANG ${ }^{1}$, SHITING HUANG ${ }^{2}$, LIANG SONG $^{1}$ and ZHOU WANG ${ }^{1}$ \\ ${ }^{1}$ Department of Thoracic Surgery, Shandong Provincial Hospital Affiliated to Shandong University; \\ ${ }^{2}$ Shandong Medical Imaging Research Institute, Shandong University, Jinan, Shandong 250021, P.R. China
}

Received July 5, 2017; Accepted December 5, 2017

DOI: 10.3892/or.2017.6145

\begin{abstract}
The esophageal squamous cell carcinoma (ESCC) subtype with STMN1 overexpression has a high likelihood of lymphatic metastatic recurrence. However, the underlying mechanism remains to be further elucidated. We assessed the expression level of STMN1 and PTEN in 96 pNO ESCC patient tissues using immunohistochemistry and western blot analysis Then, the association between STMN1 overexpression and postoperative lymphatic metastatic recurrence was evaluated. In addition, the relationship between STMN1 and PTEN was also assessed. The results showed that STMN1 expression was significantly higher in tumor tissues $(\mathrm{P}=0.013)$. STMN1 overexpression was related to tumor length $(\mathrm{P}=0.003)$ and depth of invasion $(\mathrm{P}=0.019)$. In addition, STMN1 overexpression was significantly associated with postoperative lymphatic metastatic recurrence in pN0 ESCC patients. Patients with STMN1-overexpressing tumors had a higher 3-year lymphatic metastatic recurrence rate $(\mathrm{P}=0.024)$. Furthermore, in laboratory experiments, STMN1 expression was stably silenced using lentiviral vector delivery of shRNA in Eca109 and EC9706 cell lines to assess the functional effect of STMN1 in vitro. The results indicated that stable silencing of STMN1 expression significantly inhibited cell proliferation, migration and invasion. Moreover, we inactivated the PI3K pathway in ESCC cell lines with the PI3K inhibitor LY294002 and then detected STMN1 expression by western blot analysis. STMN1 levels were robustly reduced consistent with the downregulation of p-Akt (S473) by PI3K pathway inhibition. STMN1 can act as a marker to quantitatively measure the activation of the PI3K pathway and stratify patients accordingly.
\end{abstract}

\section{Introduction}

Esophageal cancer is one of the most common upper gastrointestinal tract malignant neoplasms, and is the sixth leading

Correspondence to: Dr Zhou Wang, Department of Thoracic Surgery, Shandong Provincial Hospital Affiliated to Shandong University, 324 Jingwu Road, Jinan, Shandong 250021, P.R. China E-mail: wangzhou620226@163.com

Key words: STMN1, marker, ESCC, poor prognosis, PI3K pathway cause of cancer-related mortality worldwide (1). Esophageal squamous cell carcinoma (ESCC) accounts for more than $70 \%$ of esophageal cancers worldwide and is the main pathological type of all esophageal cancer in the Chinese population (2-4). Recently, despite advances in the systemic treatment of ESCC, the prognosis is far from satisfactory (5). In addition, the prognosis of ESCC varies widely between individuals. To stratify patients and develop personalized treatment, it is of clinical importance to elucidate the molecular mechanisms involved in the development and progression of ESCC.

Stathmin 1 (STMN1) is highly conserved and plays a critical role in the assembly and disassembly of the mitotic spindle, which is necessary in the final stage of cell division, and its mutation may lead to uncontrolled cell proliferation (6-8). This role in cell cycle regulation may classify STMN1 as an oncoprotein. It was reported that STMN1 overexpression correlates with invasion and metastasis in many human malignancies, such as lymphoma, ovarian, prostate, breast and lung cancer (9). In addition, STMN1 overexpression was also detected in ESCC. Although, a previous study has shown that STMN1 overexpression predicts high risk for lymphatic metastatic recurrence and a poor prognosis in pNO ESCC patients (10), the underlying mechanism remains unclear. A series of recent studies suggest that malfunction of signaling pathways plays an important role in promoting proliferation and invasion of ESCC cells and is associated with poor ESCC prognosis. Activation of the oncogenic phosphatidylinositol 3-kinase (PI3K) pathway is frequent in solid tumors (11-13). Multiple cellular processes that are critical for tumorigenesis, such as cell proliferation, apoptosis, migration, glucose metabolism and angiogenesis, are regulated by PI3K signaling $(14,15)$. Meanwhile, the PI3K/Akt pathway is negatively regulated by the tumor suppressor gene phosphatase and tensin homolog deleted on chromosome 10 (PTEN) (16). It is well documented that activation of PTEN/PI3K pathway signaling is a biological marker of poor prognoses in breast, prostate and bladder carcinoma (17) and it is involved in the cisplatin resistance of ESCC cells $(18,19)$. There may be a relationship between STMN1 acting as an oncoprotein and activation of the PI3K pathway.

In the present study, we investigated the correlation between the expression of STMN1 and the prognosis of pN0 ESCC patients. Moreover, we employed laboratory experiments to detect the functional effect of STMN1 on cellular 
ability related to tumor metastasis in vitro. To determine the possible underlying mechanisms of high lymphatic metastatic recurrence rate in ESCC patients with the STMN1 overexpression subtype, we performed laboratory research on the PI3K pathway to explore the possible relationships between STMN1 and the regulatory proteins involved in the PI3K pathway.

\section{Materials and methods}

Ethics statement. The study protocol was approved by the Research Ethics Committee of Shandong Provincial Hospital affiliated to Shandong University (protocol no. 2017-204). All participants provided their written informed consent for use of the tissues and data analysis.

Patients and specimens. Thirty paired samples of frozen ESCC tissues and corresponding healthy esophageal mucosa (CHEM, $>5 \mathrm{~cm}$ from the margin of ESCC) were harvested from surgical specimens in our department from January 2016 to May 2016. In addition, from December 2011 through December 2012, 113 patients with mid-thoracic ESCC who had undergone an Ivor Lewis esophagectomy with two-field lymph node dissection in our department were retrospectively studied. Patients did not receive chemotherapy or radiotherapy before surgery and all of them underwent a complete tumor resection. In addition, individuals enrolled in the present study were all restaged with stage pN0 according to postoperative pathology (American Joint Committee on Cancer Staging Manual, 7th edition). Among them, 17 patients were lost to follow-up. The remaining eligible 96 patients were enrolled in this study, and the detailed characteristics of the 96 patients are listed in Table I.

Immunohistochemistry of tissue specimens. The STMN1 and PTEN expression levels were detected by immunohistochemistry using a streptavidin-peroxidase (SP) method according to a previously published procedure (20). Rabbit anti-STMN1 (cat. no. ab52906) and anti-PTEN polyclonal (cat. no. ab170941) antibodies were respectively diluted at 1:150 and 1:50 (Abcam, Cambridge, MA, USA), and the secondary biotinylated antibody kit was purchased from Beijing ZSGB Biotechnology (Beijing, China).

All sections were examined by two independent pathologists who were blinded to the clinical data. The immunohistochemical score (IHS) was identified by combining the proportion score (percentage of positively stained cells) with the staining intensity score. For STMN1 expression, the proportion score ranges were as follows: $0(<5 \%), 1(5-24 \%)$, $2(25-49 \%), 3(50-74 \%)$ and $4(\geq 75 \%)$ while the staining intensity was scored as 0 (negative), 1 (weak), 2 (moderate) and 3 (strong). The IHS of each case was generated by multiplying the proportion score and staining intensity score. Cases with an IHS $\geq 4$ were considered to have STMN1-positive expression (10). For PTEN expression, the quantity score ranges were as follows: $0(<5 \%), 1(5-25 \%), 2(26-50 \%)$ and $3(>50 \%)$. The staining intensity was scored as: 0 (absent), 1 (weak staining), 2 (moderate staining) and 3 (strong staining). The total score was classified into negative expression (from 0 to 2 ) and positive expression (from 3 to 9) (21).
Western blot analysis. Protein was extracted from tissue specimens and cells, and the concentration was determined using a bicinchoninic acid assay. Equal amounts of protein $(40 \mu \mathrm{g})$ were electrophoresed on $10 \%$ SDS polyacrylamide gels and transferred to nitrocellulose membranes. The membranes were blocked with $5 \%$ non-fat dry milk and incubated overnight at $4^{\circ} \mathrm{C}$ with primary antibodies against STMN1, PTEN and $\beta$-actin $(1: 1,000)$. After rinsing with phosphatebuffered saline (PBS), the membranes were incubated with a secondary antibody conjugated with horseradish peroxidase (HRP) anti-rabbit IgG (1:10,000; Santa Cruz Biotechnology, Inc., Santa Cruz, CA, USA). The protein levels were quantified using an enhanced chemiluminescence (ECL) detection system (Amersham Imager 600; GE Healthcare, Chicago, IL, USA).

Cell culture, treatment and transfection. The human ESCC cell lines (Eca109 and EC9706) were purchased from the Cell Bank of Shanghai Institute in China (Shanghai, China). All cells were maintained in RPMI-1640 medium supplemented with $10 \%$ fetal bovine serum (FBS) and $1 \%$ penicillin/streptomycin (both from HyClone Laboratories, Inc., Logan, UT, USA). These cells were grown at $37^{\circ} \mathrm{C}$ in an atmosphere of $5 \% \mathrm{CO}_{2}$.

The PI3K-inhibitor LY294002 was purchased from Selleck Chemicals (Houston, TX, USA). Cells were cultured in LY294002 $(20 \mu \mathrm{M})$ for 4 days to inhibit the activation of the PI3K pathway.

Human short hairpin RNA (shRNA) was synthesized and packaged in Open Biosystems (Thermo Fisher Scientific, Inc., Waltham, MA, USA). The targeting sequence of STMN1 was 5'-TTATTAGCTTCCATTTTGT-3' and 5'-TTATTAACC ATTCAAGTCC-3' (22). Eca109 and EC9706 cell lines were transfected with the lentivirus-mediated shRNA according to the manufacturer's instructions. The multiplicity of infection (MOI) was 20 for Eca109 and 30 for EC9706 cells. A normal control (NC) shRNA was used as a blank control. Puromycin at a concentration of $5 \mu \mathrm{g} / \mathrm{ml}$ was used to select the transfected cells. qRT-PCR and western blot analysis were used to determine the transfection efficiency.

Immunocytochemistry of cell lines. Eca109 and EC9706 cells were seeded into 4-chambered glass slides (Nunc Lab-Tek Chamber Slide System; Thermo Fisher Scientific) and incubated overnight. After rinsing with PBS, cells were fixed with $3.7 \% \mathrm{w} / \mathrm{v}$ paraformaldehyde (Sigma-Aldrich, St. Louis, MO, USA). Then, cells were rinsed with PBS and permeabilized in $0.5 \%$ Triton X-100 (Sigma-Aldrich). Five percent normal goat serum and $0.5 \%$ NP-40 (Sigma-Aldrich) were used to block the non-specific immunoglobulin binding. After incubation with the primary antibody diluted 1:100 in blocking solution, cells were rinsed with $0.05 \%$ Tween-20 (Bio-Rad Laboratories, Hercules, CA, USA) in PBS and incubated with secondary antibody for $1 \mathrm{~h}$ at room temperature. Then, cells were stained with 3,3'-diaminobenzidine (DAB) and observed under a light microscope.

$R N A$ extraction and $q R T-P C R$. Total RNA was extracted from the cells using TRIzol reagent (Invitrogen, Carlsbad, CA, USA). The procedures for RNA extraction and qRT-PCR were 
Table I. Correlations between STMN1 expression and the clinicopathological factors of the pN0 ESCC cases.

\begin{tabular}{|c|c|c|c|c|c|c|}
\hline \multirow[b]{2}{*}{ Clinical characteristics } & \multirow[b]{2}{*}{$\begin{array}{c}\text { Patients } \\
(\mathrm{N}=96)\end{array}$} & \multicolumn{2}{|c|}{ STMN1 expression } & \multirow[b]{2}{*}{ P-value ${ }^{a}$} & \multirow[b]{2}{*}{ Recurrence rate $(\%)$} & \multirow[b]{2}{*}{ P-value ${ }^{b}$} \\
\hline & & $\begin{array}{c}(-) \\
(n=43)\end{array}$ & $\begin{array}{c}(+) \\
(\mathrm{n}=53)\end{array}$ & & & \\
\hline $\operatorname{Sex}$ & & & & 0.943 & & 0.821 \\
\hline Male & 74 & 33 & 41 & & $28(38.9)$ & \\
\hline Female & 22 & 10 & 12 & & $9(40.9)$ & \\
\hline Age (years) & & & & 0.958 & & 0.434 \\
\hline$<50$ & 36 & 16 & 20 & & $12(33.3)$ & \\
\hline$\geq 50$ & 60 & 27 & 33 & & $25(41.7)$ & \\
\hline Tumor length $(\mathrm{cm})$ & & & & 0.003 & & 0.123 \\
\hline$<3$ & 44 & 27 & 17 & & $14(31.8)$ & \\
\hline$\geq 3$ & 52 & 16 & 36 & & $26(50)$ & \\
\hline Differentiation & & & & 0.658 & & 0.016 \\
\hline Well + moderate & 64 & 26 & 38 & & $21(32.8)$ & \\
\hline Poor & 32 & 17 & 15 & & $16(50)$ & \\
\hline pT & & & & 0.019 & & 0.002 \\
\hline $\mathrm{T} 1$ & 8 & 5 & 3 & & $0(0)$ & \\
\hline $\mathrm{T} 2$ & 37 & 22 & 15 & & $9(24.3)$ & \\
\hline $\mathrm{T} 3$ & 51 & 16 & 35 & & $28(54.9)$ & \\
\hline PTEN expression & & & & 0.001 & & 0.026 \\
\hline$(-)$ & 51 & 15 & 36 & & $25(49.0)$ & \\
\hline$(+)$ & 45 & 28 & 17 & & $12(26.7)$ & \\
\hline
\end{tabular}

${ }^{\mathrm{a} C h i}$-square test; ${ }^{\mathrm{b}} \mathrm{P}$-value log-rank test.

detailed as described in our previous study (20). The sequences of primers used were as follows: STMN1 forward 5'-AGAATA CACTGCCTGTCGCTTG-3' and reverse 5'-AGGCACGCT TCTCCAGTT-3'; $\beta$-actin forward 5'-TGGAGAAAATCT GGCACCAC-3' and reverse 5'-GGTCTCAAACATGATCTG G-3' (22). The relative expression levels were normalized to endogenous $\beta$-actin expression.

Cell proliferation assay. In the cell proliferation assay using a Cell Counting Kit-8 (CCK-8; Dojindo Laboratories, Kymamoto, Japan), viable cells were seeded into 96-well tissue culture plates such that there were 3,000 cells in a final volume of $100 \mu \mathrm{l} /$ well. Every $24 \mathrm{~h}, 10 \mu \mathrm{l}$ of CCK- 8 solution was added to each well and then the plate was incubated for $4 \mathrm{~h}$ at $37^{\circ} \mathrm{C}$. The viable cells were identified by absorbance measurements at $450 \mathrm{~nm}$ using a microplate reader (Bio-Rad Laboratories). The experiment was performed in triplicate.

Migration and invasion assays. For the Transwell migration and invasion assays, cells were pre-cultured in serum-free medium for $24 \mathrm{~h}$. In addition, $1.5 \times 10^{5}$ cells in $200 \mu \mathrm{l}$ serum-free medium were seeded into upper chambers of a 24-well Transwell apparatus (8- $\mu \mathrm{m}$ pore size; Merck Millipore, Darmstadt, Germany). Six hundred microliters of medium with $15 \%$ FBS was added to the lower chambers. After incubation for $24 \mathrm{~h}$, cells remaining in the upper chambers were removed by scraping. Cells that had migrated through the membrane were fixed and stained with hematoxylin and eosin (H\&E). Then, average numbers of cells per visual field were counted under a light microscope (Leica DM 4000B; Leica Microsystems, Wetzlar, Germany).

For the invasion assay, $40 \mu \mathrm{l}$ of Matrigel (BD Biosciences, San Jose, CA, USA) was diluted 1:4 in serum-free medium and used to pre-coat the upper chambers of the Transwell apparatus and left to solidify for $1 \mathrm{~h}$. Then, pre-cultured cells in $200 \mu \mathrm{l}$ of serum-free medium were added to the upper chambers. The remaining procedures were the same as the migration assay, except the duration of incubation was $48 \mathrm{~h}$.

Clonogenic assay. Transfected cells which were trypsinized to generate a single cell suspension were seeded in 6-well plates at 500 cells/well. After 14 days, the number of colonies that were stained with crystal violet and contained at least 50 cells was counted. The colony survival fraction was calculated for each treatment.

Statistical methods. The quantitative data are expressed as the mean \pm standard deviation (SD). Differences among multiple groups were analyzed using two-tailed Student's t-tests using GraphPad Prism 5 software (GraphPad Software, Inc., La Jolla, CA, USA). The Mann-Whitney U test was chosen to identify the differences in STMN1 expression as detected by immunohistochemistry. A Chi-square test was employed to analyze the correlations between the STMN1 overexpression 


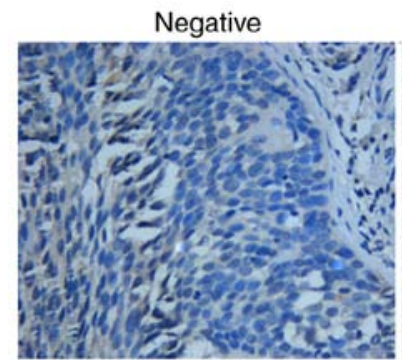

Moderate

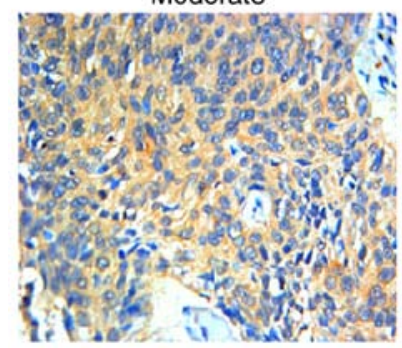

$\times 400$

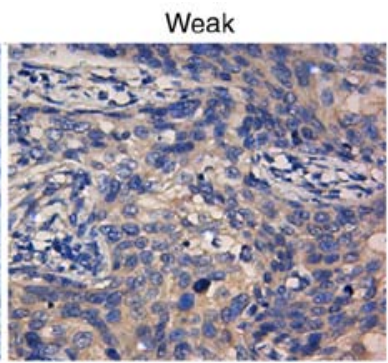

Strong

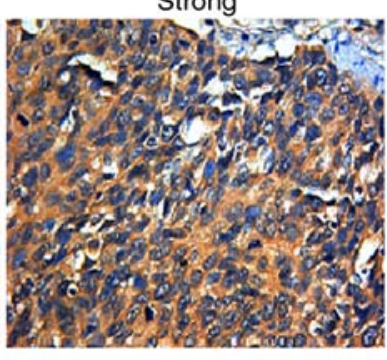

$\times 400$
Figure 1. Immunohistochemical staining of STMN1 in tumor specimens via immunohistochemistry (original magnification, $\mathrm{x} 400$ ).

and the clinicopathological factors, and those between STMN1 expression and PTEN expression. A univariate analysis was performed by modeling Kaplan-Meier survival curves. The log-rank test was used to calculate the survival rate. A multivariate analysis was carried out using the Cox proportional hazards model. Differences were considered significant when $\mathrm{P}<0.05$. The statistical data were obtained using the SPSS software package (SPSS 17.0; SPSS, Inc., Chicago, IL, USA).

\section{Results}

The expression of STMN1 in ESCC tissues and CHEM. STMN1 protein expression levels in 30 pairs of tissue specimens (ESCC tissue and CHEM) were investigated by IHC and western blot analysis. The IHC results showed that the positive expression of STMN1 was detected as a yellow or brownish yellow stain in the cytoplasm (Fig. 1). The IHC staining demonstrated that the expression level of STMN1 in ESCC was significantly higher than that in CHEM ( $\mathrm{P}=0.013$; Fig. 2). STMN1 overexpression was found in 16 cases $(53.3 \%)$ of ESCC tissues and 4 cases (13.3\%) of CHEM. Moreover, we randomly selected 8 pairs of tissue specimens (ESCC and CHEM) to confirm the STMN1 protein level by western blot analysis (Fig. 3A). Higher STMN1 protein expression was identified in tumor tissues (STMN1/ $\beta$-actin: $0.76 \pm 0.10$ vs. $0.49 \pm 0.18, \mathrm{P}=0.0021$; Fig. $3 \mathrm{~B}$ ). The results corresponded to those of the immunohistochemistry analysis.

STMN1 expression is correlated with clinical characteristics and with PTEN expression. The clinicopathological data of the 96 eligible patients with mid-thoracic ESCC were retrospectively studied. ESCC tissues from 53 patients were identified with STMN1 overexpression. The diagnostic sensitivity was $55.2 \%$ (53/96) (Table I).

By immunohistochemistry, positive expression of PTEN was detected mainly in the cytoplasm (Fig. 4) and rarely in the nuclei (only 4 stained heterogeneously). Forty-five tumor

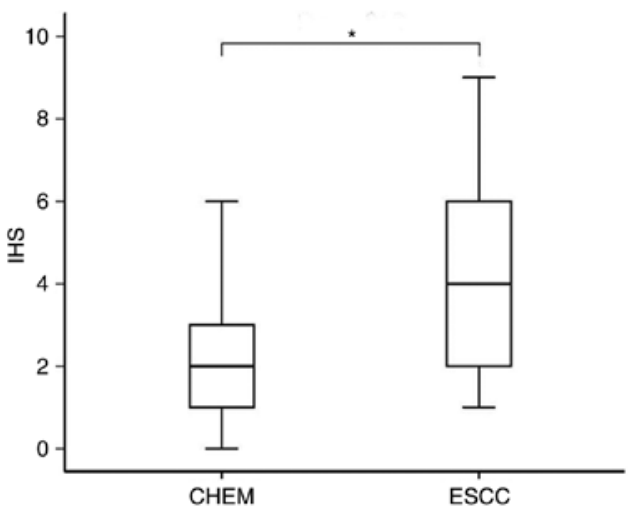

Figure 2. Immunohistochemical score (IHS) of STMN1 in esophageal squamous cell carcinoma (ESCC) and corresponding healthy esophageal mucosa (CHEM). ${ }^{*} \mathrm{P}<0.05$.

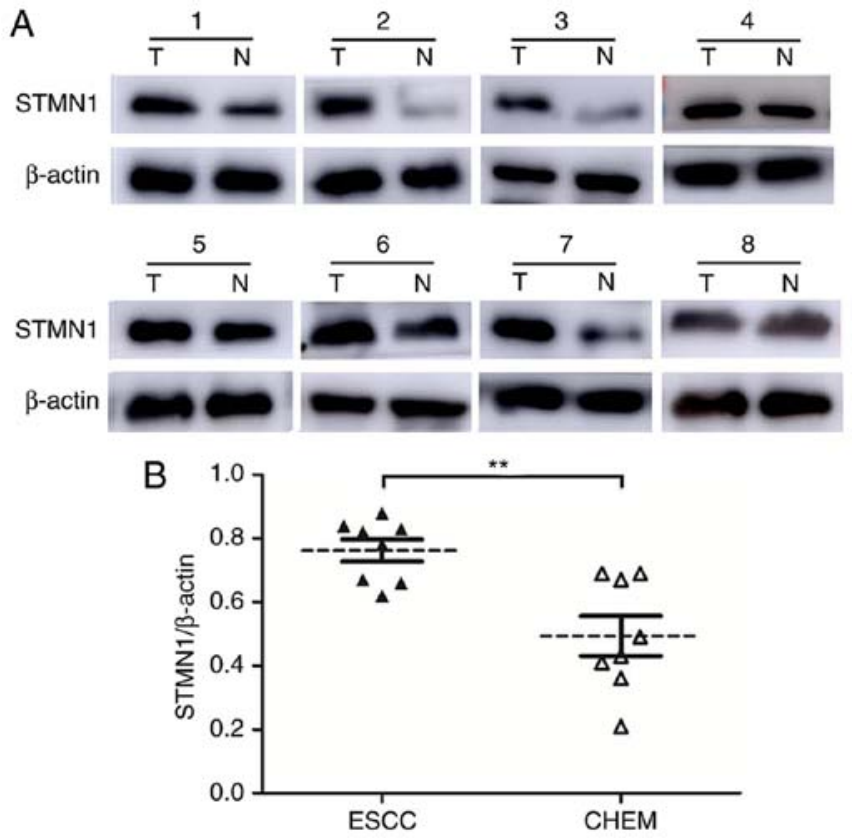

Figure 3. The expression of STMN1 protein in tissue specimens via western blot analysis. (A) The bands of STMN1 and $\beta$-actin in 8 pairs of esophageal squamous cell carcinoma (ESCC) and corresponding healthy esophageal mucosa (CHEM). (B) Quantitative analysis of STMN1 expression in ESCC and $\mathrm{CHEM}$ normalized to $\beta$-actin expression. $\mathrm{T}$, cancerous tissue; $\mathrm{N}$, corresponding healthy esophageal mucosa. The results are expressed as the mean \pm standard deviation. ${ }^{* *} \mathrm{P}<0.01$.

samples (46.9\%) showed positive expression of PTEN, whereas $51(53.1 \%)$ tumor samples showed negative expression of PTEN.

The correlation between STMN1 expression and clinicopathological features is shown in Table I. The Chi-square analysis indicated that STMN1 overexpression was significantly associated with tumor length $(\mathrm{P}=0.003)$ and depth of invasion $(\mathrm{P}=0.019)$. In addition, STMN1 expression was inversely correlated with PTEN expression $(\mathrm{P}=0.001)$. No other clinicopathological parameter was associated with STMN1 overexpression.

STMN1 expression is correlated with the overall survival and lymphatic metastatic recurrence. Through the follow-up, a first lymph node metastatic recurrence within 3 years was 
Table II. Cox regression analysis for risk factors of 3-year lymphatic metastatic recurrence in pNO ESCC patients.

\begin{tabular}{lcccccc}
\hline & B & SE & Wald & P-value & HR & $95 \%$ CI \\
\hline Sex & 0.422 & 0.422 & 1.000 & 0.317 & 0.656 & $0.287-1.500$ \\
Age (years) & 0.022 & 0.373 & 0.003 & 0.954 & 1.022 & $0.492-2.125$ \\
Tumor length & -0.122 & 0.391 & 0.097 & 0.755 & 0.885 & $0.411-1.906$ \\
T stage & 0.980 & 0.419 & 5.461 & 0.019 & 2.664 & $1.171-6.060$ \\
Differentiation & 0.676 & 0.359 & 3.542 & 0.060 & 1.967 & $0.972-3.977$ \\
STMN1 expression & 0.450 & 0.391 & 1.325 & 0.250 & 1.568 & $0.729-3.374$ \\
PTEN expression & 0.143 & 0.395 & 0.131 & 0.717 & 0.867 & $0.399-1.880$ \\
\hline
\end{tabular}

B, regression coefficient; SE, standard error; Wald, Wald value; HR, hazard ratio; CI, confidence interval.
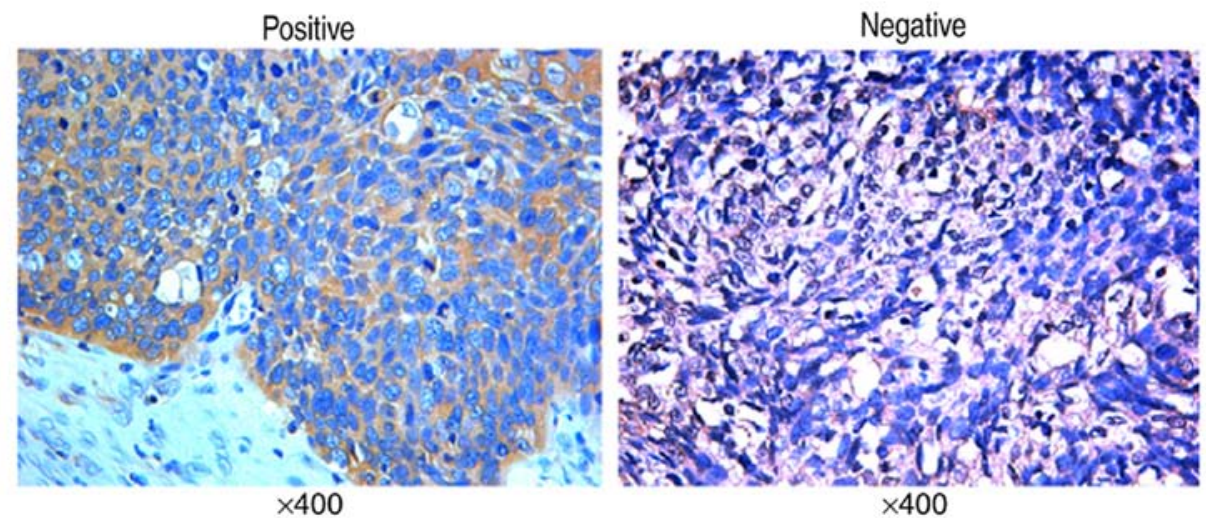

Figure 4. Representative immunohistochemical staining of PTEN in esophageal squamous cell carcinoma (ESCC) tissue and corresponding healthy esophageal mucosa (CHEM) (original magnification, x400).
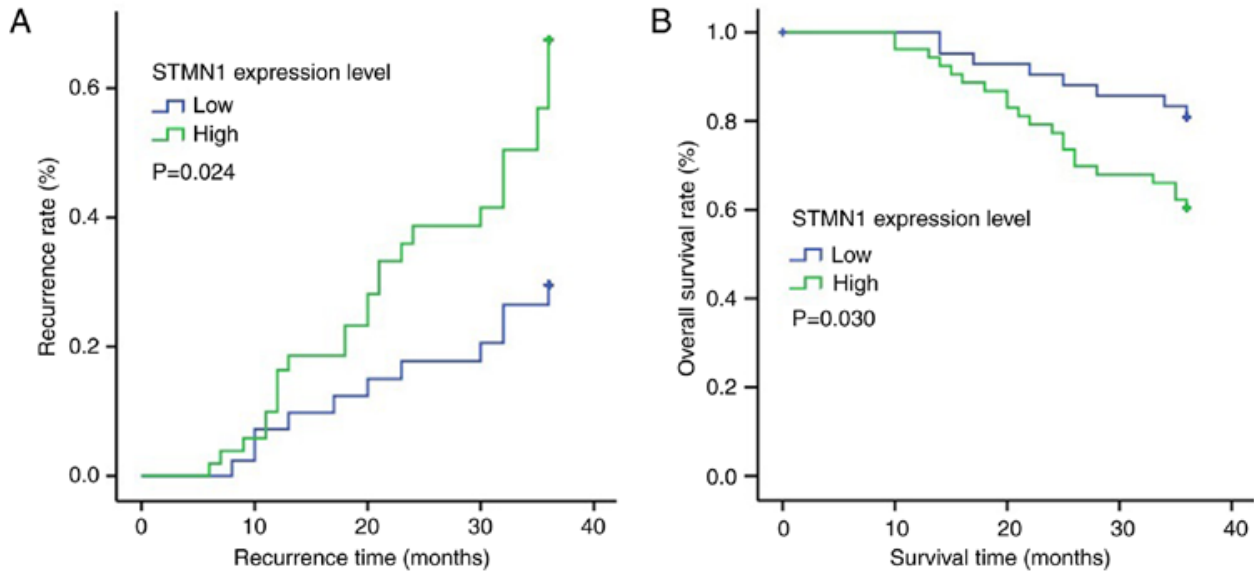

Figure 5. Kaplan-Meier analysis and log-rank test of STMN1 expression for lymphatic metastatic recurrence (A) and overall survival (B).

identified in 37 cases $(38.5 \%)$, in which STMN1 overexpression was detected in 26 patients $(70.3 \%)$. In the group with low STMN1 expression, the 3-year lymphatic metastatic recurrence rate was only $25.6 \%$ (11/43), whereas in the group with STMN1 overexpression, this rate reached 49.1\% (26/53). The Kaplan-Meier analysis and the log-rank test showed that patients with STMN1 overexpression had a significantly higher lymphatic metastatic recurrence rate (Fig. $5 \mathrm{~A} ; \mathrm{P}=0.024)$ and a significantly worse overall survival rate (Fig. 5B; $\mathrm{P}=0.030$ ).
In addition, $\mathrm{T}$ stage $(\mathrm{P}=0.002)$, differentiation degree $(\mathrm{P}=0.016)$ and $\mathrm{PTEN}$ levels $(\mathrm{P}=0.026)$ were also indicated to be associated with lymphatic recurrence in pNO ESCC patients by univariate analysis (Table I). The Cox proportional hazards model was performed to identify factors involved in lymphatic recurrence of pN0 ESCC patients. The Cox multivariate regression analysis revealed that the $\mathrm{T}$ stage $(\mathrm{P}=0.019)$ and the tumor differentiation degree $(\mathrm{P}=0.060)$ were both independent prognostic factors for pNO ESCC (Table II). 


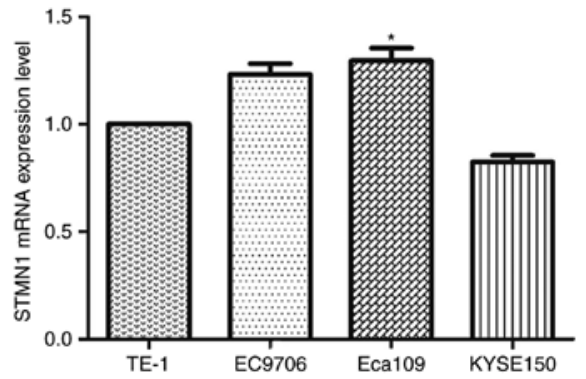

Figure 6. Relative expression level of STMN1 mRNA in four ESCC cell lines via qRT-PCR. "P<0.05 compared to TE-1.

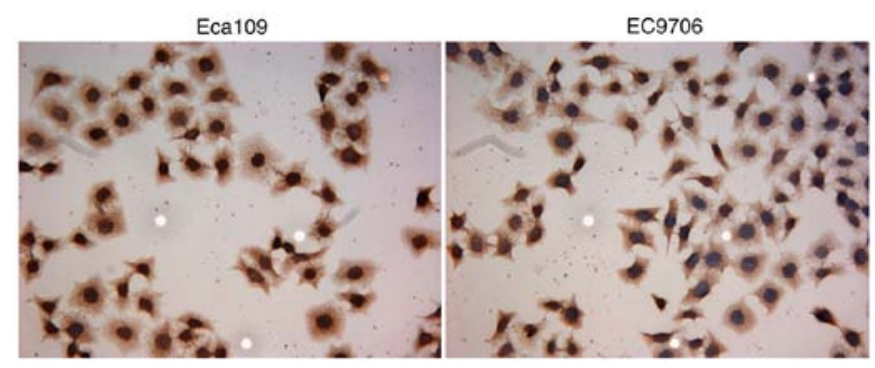

Figure 7. Immunohistochemical staining of STMN1 in Eca109 and EC9706 cells.

Expression of STMN1 in cell lines. qRT-PCR was performed to evaluate the expression of STMN1 in four esophageal cancer cell lines: Eca109, KYSE150, TE-1 and EC9706. Eca109 and EC9706 showed relatively high expression of STMN1 (Fig. 6). Immunocytochemistry was used to verify the result of qRT-PCR. By immunocytochemistry, the positive expression of STMN1 protein showed a yellow or brownish yellow stain in the cytoplasm of tumor cells (Fig. 7). Strong immunoreactivity of STMN1 protein was detected in the cytoplasm of Eca109 and EC9706 cells. Therefore, Eca109 and EC9706 were selected as the candidate cell line for shRNA transfection.

Lentiviral-mediated shRNA silencing of STMN1 gene expression. After transfection, western blot analysis was used to confirm the efficacy. The results showed that the expression of the STMN1 protein was significantly downregulated in the lentiviral-mediated STMN1 shRNA-transfected Eca109 and EC9706 cells $(\mathrm{P}<0.001$; Fig. 8). There was no significant difference in STMN1 expression between the NC shRNA (control) group and the untransfected group. These results showed that stable transfection of STMN1 shRNA can effectively and specifically silence STMN1 gene expression.

Stable silencing of STMN1 inhibits cell proliferation. The CCK-8 assay showed that the cell growth rate of Eca109 and EC9706 cells transfected with STMN1 shRNA was significantly lower compared to those cells transfected with NC shRNA. The OD450 values of the Eca109 and EC9706 cells transfected with STMN1 shRNA were significantly decreased at 24, 48 and $72 \mathrm{~h}(\mathrm{P}<0.01$; Fig. 9A and $\mathrm{B})$. The clonogenic assay (Fig. 9C) showed that the colony numbers of Eca109 and EC9706 cells transfected with STMN1
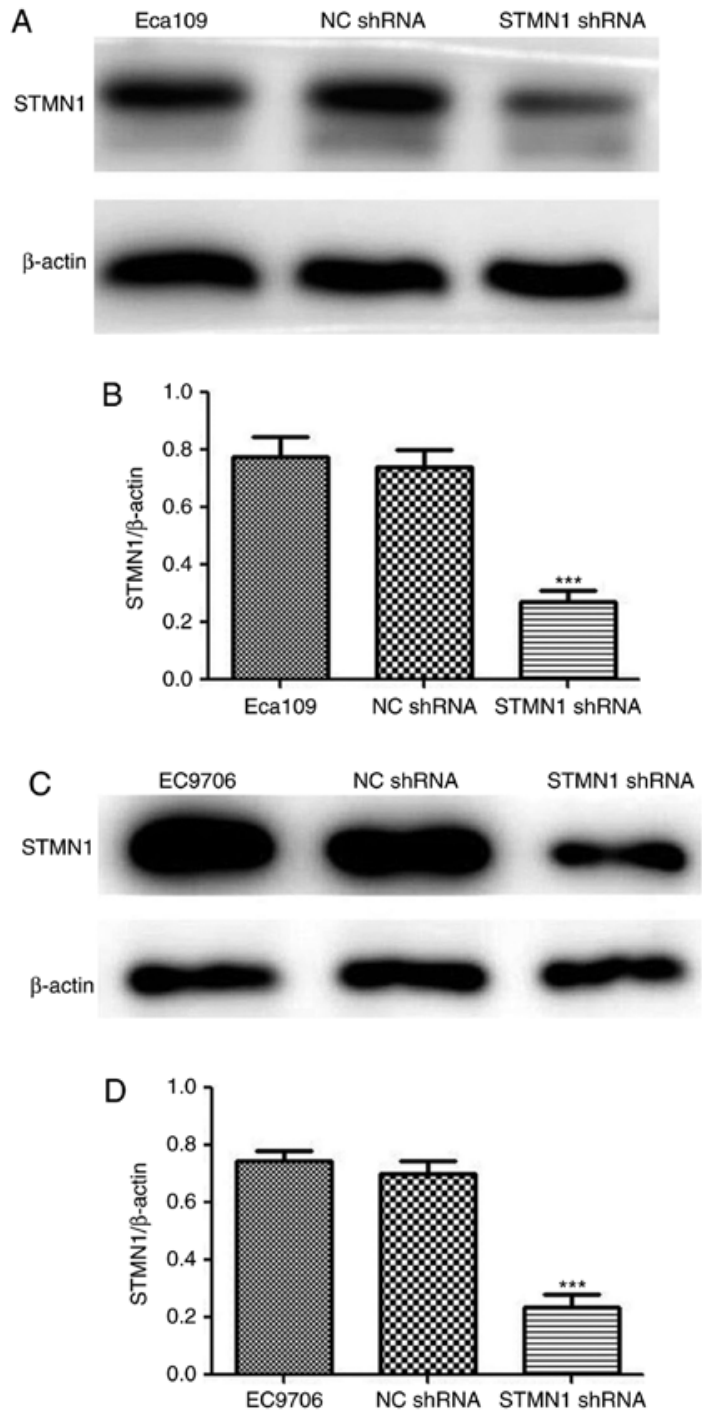

Figure 8. Bands and quantitative analysis of STMN1 protein in untransfected, NC shRNA and STMN1 shRNA groups via western blot analysis. The results are expressed as the mean \pm standard deviation. ${ }^{* * *} \mathrm{P}<0.001$ compared to NC shRNA.

shRNA were significantly less than those transfected with NC shRNA $(\mathrm{P}<0.05$; Fig. 9D and $\mathrm{E})$. These results indicated that stable silencing of STMN1 may inhibit the cell proliferation.

Stable silencing of STMN1 inhibits cell migration and invasion. Migration and invasion assays were used to test the effect of STMN1 on cell motility and invasion. The migration assay showed that the number of migrated cells in the STMN1 shRNA group were significantly decreased in the lower chamber compared with cells in the NC shRNA group $(\mathrm{P}<0.0001$; Fig. 10A). At the same time, the number of invaded cells in the STMN1 shRNA group was also decreased compared to that in NC shRNA group in the invasion assay $(\mathrm{P}<0.0001$; Fig. 10B and C). These results indicated that stable silencing of STMN1 may inhibit the invasive and metastatic ability of ESCC cells.

STMN1 is PI3K pathway-regulated in ESCC cells in vitro. To identify the possible relationship between STMN1 and 
A

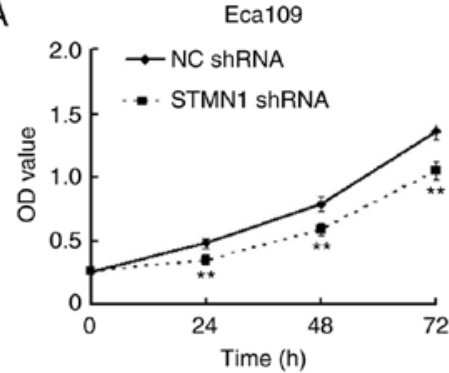

B

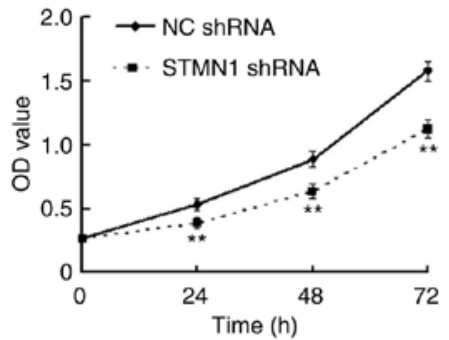

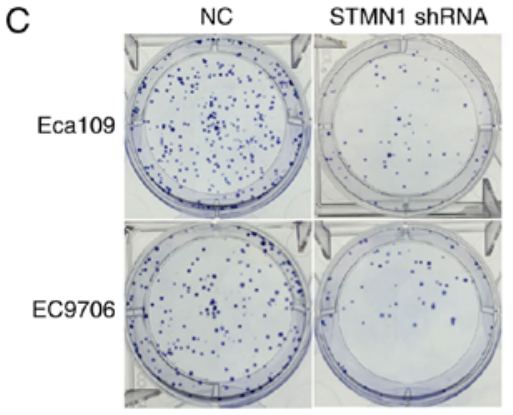
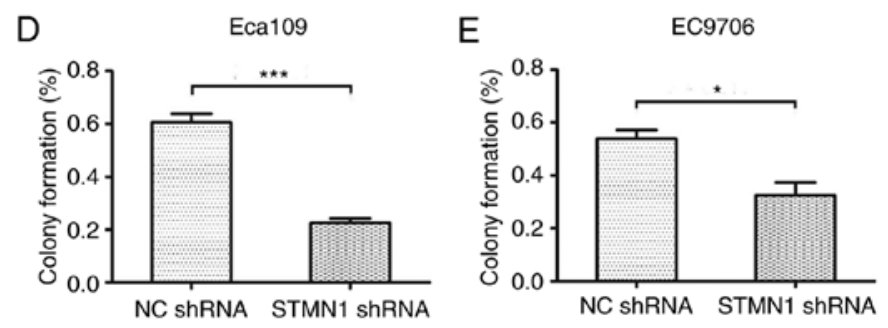

Figure 9. Proliferative ability was decreased in the STMN1 shRNA groups. (A and B) The OD450 values were significantly decreased in the STMN1 shRNA groups. (C-E) The colony formation rates were reduced in the STMN1 shRNA groups. The results are expressed as the mean \pm standard deviation. ${ }^{*}<0.05$, ${ }^{* * *} \mathrm{P}<0.01,{ }^{* * *} \mathrm{P}<0.001$

A
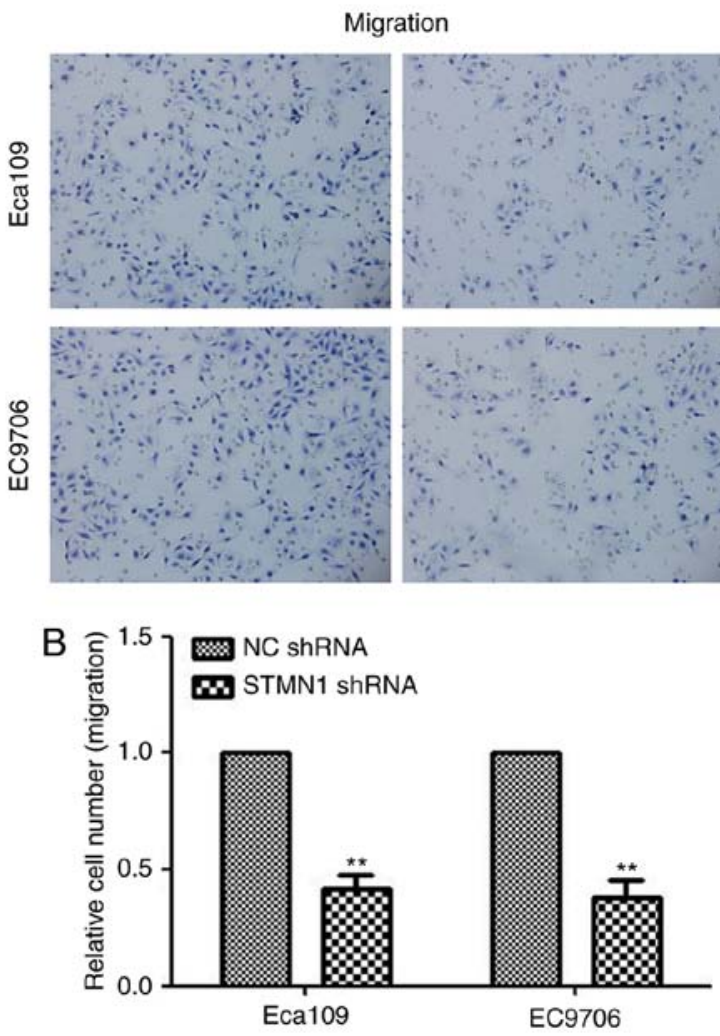

\section{igration}
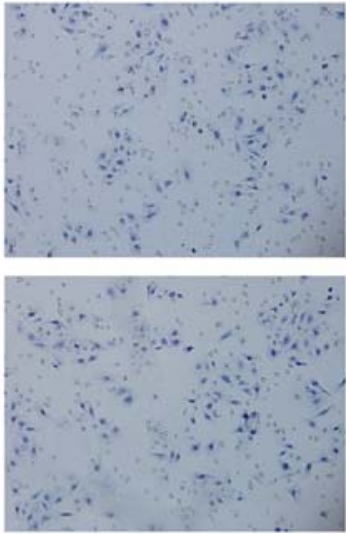

ECI706
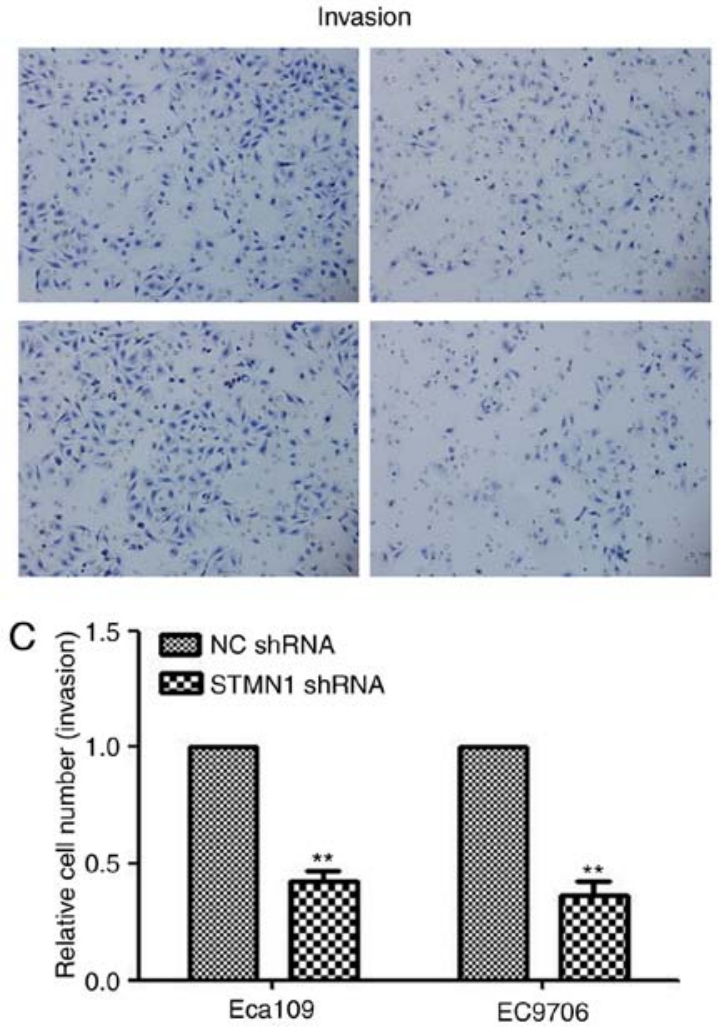

Figure 10. Migration and invasion abilities were decreased in the STMN1 shRNA groups. The Transwell assays (A) conducted in the Eca109 and EC9706 cells showed that the migrated and invaded cell numbers were reduced in the STMN1 shRNA groups (B and C). The results are expressed as the mean \pm standard deviation. ${ }^{* *} \mathrm{P}<0.01$ compared to NC shRNA.

the activation of the PI3K pathway, we detected the status of p-Akt (S473) to identify the activation of the PI3K pathway in STMN1 shRNA Eca109 cells. Western blot analysis showed that there was no obvious change in p-Akt expression after STMN1 was silenced (Fig. 11A).
Then, we treated Eca109 and EC9706 cells with LY294002 (diluted in DMSO) to inhibit the activation of the PI3K pathway and then detect STMN1 expression by western blot analysis. The results showed that STMN1 levels were robustly reduced, consistent with the downregulation of p-Akt (S473) by PI3K 
A
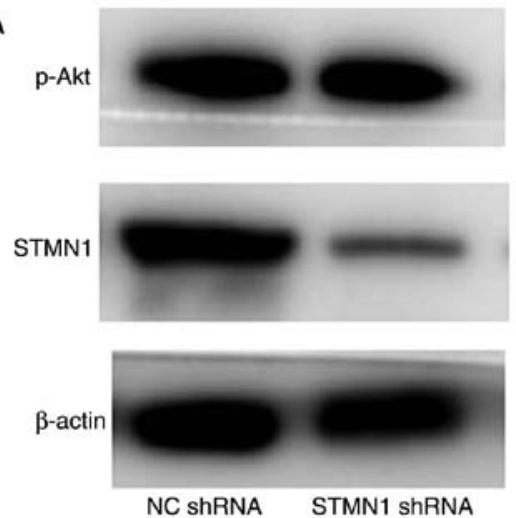

B
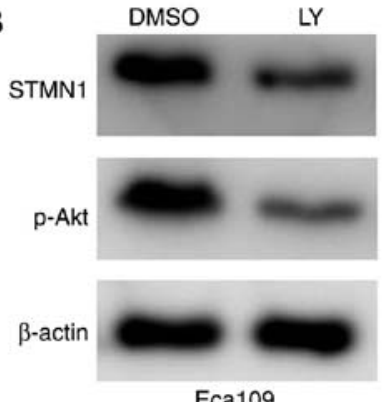

Eca109
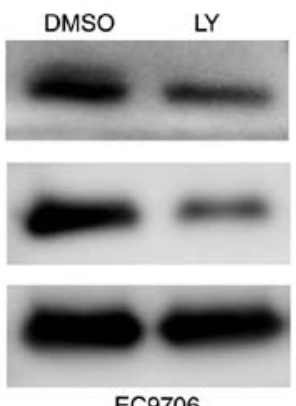

EC9706

Figure 11. (A) Bands of p-Akt and $\beta$-actin in STMN1 shRNA-transfected Eca109 cells. (B) Expression of STMN1 in Eca109 and EC9706 cells treated with DMSO (negative control) or the PI3K pathway inhibitor LY294002. LY, LY294002.

pathway inhibition both in the Eca109 and EC9706 cell line (Fig. 11B).

\section{Discussion}

Currently, postoperative adjuvant therapy is not recommended in pNO ESCC after radical operation according to the NCCN guidelines. However, multiple ESCC patients in the pN0 stage tend to relapse after R0 resection, and the common type of relapse is lymph node metastatic recurrence. With surgery alone, the 5-year survival rate of ESCC patients in stage IB and IIA is 62 and $55 \%$, respectively $(23,24)$. The prognosis of ESCC is far from satisfactory even in $\mathrm{pN} 0$ stage. Also, it is of clinical importance to predict lymphatic metastatic recurrence early and select those candidates to undergo postoperative adjuvant therapy.

STMN1 is a ubiquitously expressed phosphoprotein. It plays a critical role in the assembly and disassembly of the mitotic spindle, which is necessary in the final stage of cell division. In addition, it regulates cell cycle progression by influencing the dynamics of microtubules $(25,26)$. Its role in regulating the cell cycle makes STMN1 act as an oncoprotein. Overexpression of STMN1 in human cancer was reported to be associated with malignancy and poor prognosis (27). It was demonstrated that STMN1 is overexpressed in ESCC, and STMN1 overexpression predicts a high risk for lymphatic metastatic recurrence in pNO ESCC patients (10). However, the functional effect of STMN1 in vitro was not elucidated. It is well known that the abilities of primary tumor cells to invade and propagate are vital factors that are involved in tumor metastasis (28). In the present study, we used shRNA to silence the expression of STMN1 in ESCC cell lines to study the effect of STMN1 on cellular ability related to tumor metastasis in vitro.

Eca109 and EC9706 cells were chosen to analyze the effect of STMN1 suppression by shRNA due to their higher expression of STMN1. After stable transfection, western blot analysis showed that expression of STMN1 in Eca109 and EC9706 was stably suppressed. The CCK-8 assay showed that suppressing STMN1 expression significantly inhibited the growth rates of the Eca109 and EC9706 cells. Migration and invasion assays confirmed that knockdown of STMN1 significantly inhibited the abilities of motility and invasion in Eca109 and EC9706 cells. We concluded that the STMN1 expression was associated with the ability for metastasis in vitro in ESCC.
Despite the fact that STMN1 has proven to act as a tumor signature that is capable of discriminating ESCC patients with good or poor outcomes, deciphering the biological basis of why it is predictive remains a significant challenge. The activation of the oncogenic PI3K pathway is frequent in solid tumors. In addition, it was estimated that aberrant PI3K pathway signaling is present in more than $30 \%$ of human cancers (29). The PI3K pathway is involved in many aspects of tumor biology: cell transformation, growth, proliferation, migration, protection from apoptosis, genomic instability, angiogenesis and metastasis (29,30). Akt, a small family of serine/threonine protein kinases, is the end-point of the PI3K pathway. Activated Akt can phosphorylate a number of downstream substrates to regulate the above cellular processes (31). Conversely, the lipid phosphatase PTEN can dephosphorylate the 3'-end of phosphatidylinositols so that it can attenuate Akt activation and negatively regulate the PI3K pathway (32). Despite the consensus that activation of PI3K signaling would confer an aggressive tumor phenotype, there remains a lack of markers that can predict pathway activation and actual patient outcomes (33-35). Theoretically, phosphorylated Akt (p-Akt) at serine 473 is the most reliable marker of PI3K pathway activation. However, it plays a role only in highly controlled situations, such as in cell culture. The available reagents do not work well in routine clinical specimens. Therefore, there are limitations to indentifying activation of the PI3K pathway by p-Akt in the clinical setting.

To study the possible relationship between STMN1 and the PI3K pathway, we detected the expression of STMN1 and PTEN in ESCC tissues. The expression of STMN1 was inversely correlated with that of PTEN. Meanwhile, as the most reliable PI3K pathway marker under highly controlled situations is p-Akt at serine 473, we detected the status of p-Akt in Eca109 and EC9706 cells in which STMN1 was silenced by shRNA. The western blot analysis revealed that there were no significant changes in p-Akt expression after STMN1 was stably silenced. Subsequently, we treated the Eca109 and EC9706 cells with LY294002 to inhibit activation of the PI3K pathway and then detect the expression of STMN1. The results showed that STMN1 levels were robustly reduced, consistent with downregulation of p-Akt (S473) by PI3K pathway inhibition. Therefore, we concluded that STMN1 is PI3K pathway-regulated in vitro in ESCC. Thus, STMN1 can 
act as a marker to quantitatively measure the activation of the PI3K pathway and stratify patients accordingly.

The limitation of the present study is that the functional effect of STMN1 on the cellular ability related to tumor metastasis was determined only in vitro, and the in vivo effect was not included. In future research, we will enroll xenograft tumor models in nude mice to study the functional effect of STMN1 in ESCC in vivo.

In conclusion, STMN1 overexpression was significantly associated with lymphatic metastatic recurrence in pNO ESCC patients. STMN1 levels are regulated by the PI3K pathway, and STMN1 can act as a surrogate marker of PI3K pathway signaling related to tumor recurrence. STMN1 may be clinically useful to select patients for PI3K pathway-targeted therapy and to monitor the therapeutic efficacy in ESCC.

\section{Acknowledgements}

The present study was funded by the Science and Technology Development Plan Project of Shandong Province (grant no. 2014GSF118167).

\section{References}

1. Napier KJ, Scheerer M and Misra S: Esophageal cancer: A review of epidemiology, pathogenesis, staging workup and treatment modalities. World J Gastrointest Oncol 6: 112-120, 2014.

2. Holmes RS and Vaughan TL: Epidemiology and pathogenesis of esophageal cancer. Semin Radiat Oncol 17: 2-9, 2007.

3. Dawsey SM, Lewin KJ, Liu FS, Wang GQ and Shen Q: Esophageal morphology from Linxian, China. Squamous histologic findings in 754 patients. Cancer 73: 2027-2037, 1994.

4. Cook MB: Non-acid reflux: The missing link between gastric atrophy and esophageal squamous cell carcinoma? Am J Gastroenterol 106: 1930-1932, 2011.

5. Zhang Y: Epidemiology of esophageal cancer. World J Gastroenterol 19: 5598-5606, 2013.

6. Doye V, Le Gouvello S, Dobransky T, Chneiweiss H, Beretta L and Sobel A: Expression of transfected stathmin cDNA reveals novel phosphorylated forms associated with developmental and functional cell regulation. Biochem J 287: 549-554, 1992.

7. Jeon TY, Han ME, Lee YW, Lee YS, Kim GH, Song GA, Hur GY, Kim JY, Kim HJ, Yoon S, et al: Overexpression of stathmin1 in the diffuse type of gastric cancer and its roles in proliferation and migration of gastric cancer cells. Br J Cancer 102: 710-718, 2010.

8. Bieche I, Lachkar S, Becette V, Cifuentes-Diaz C, Sobel A, Lidereau R and Curmi PA: Overexpression of the stathmin gene in a subset of human breast cancer. Br J Cancer 78: 701-709, 1998.

9. Rana S, Maples PB, Senzer N and Nemunaitis J: Stathmin 1: A novel therapeutic target for anticancer activity. Expert Rev Anticancer Ther 8: 1461-1470, 2008.

10. Akhtar J, Wang Z, Jiang WP, Bi MM and Zhang ZP: Stathmin overexpression identifies high risk for lymphatic metastatic recurrence in pNO esophageal squamous cell carcinoma patients. J Gastroenterol Hepatol 29: 944-950, 2014.

11. Altomare DA and Testa JR: Perturbations of the AKT signaling pathway in human cancer. Oncogene 24: 7455-7464, 2005.

12. Vivanco I and Sawyers CL: The phosphatidylinositol 3-kinase AKT pathway in human cancer. Nat Rev Cancer 2: 489-501, 2002.

13. Carnero A: The PKB/AKT pathway in cancer. Curr Pharm Des 16: 34-44, 2010.

14. Datta SR, Brunet A and Greenberg ME: Cellular survival: A play in three Akts. Genes Dev 13: 2905-2927, 1999.
15. Vanhaesebroeck B and Waterfield MD: Signaling by distinct classes of phosphoinositide 3-kinases. Exp Cell Res 253: 239-254, 1999.

16. Shi Y, Paluch BE, Wang X and Jiang X: PTEN at a glance. J Cell Sci 125: 4687-4692, 2012.

17. Saal LH, Johansson P, Holm K, Gruvberger-Saal SK, She QB, Maurer M, Koujak S, Ferrando AA, Malmström P, Memeo L, et al: Poor prognosis in carcinoma is associated with a gene expression signature of aberrant PTEN tumor suppressor pathway activity. Proc Natl Acad Sci USA 104: 7564-7569, 2007.

18. Chen J, Lan T, Zhang W, Dong L, Kang N, Fu M, Liu B, Liu K, Zhang C, Hou J and Zhan Q: Dasatinib enhances cisplatin sensitivity in human esophageal squamous cell carcinoma (ESCC) cells via suppression of PI3K/AKT and Stat 3 pathways. Arch Biochem Biophys 575: 38-45, 2015.

19. $\mathrm{Xu} \mathrm{J}$ and $\mathrm{Hu} \mathrm{Z}$ : Y-box-binding protein 1 promotes tumor progression and inhibits cisplatin chemosensitivity in esophageal squamous cell carcinoma. Biomed Pharmacother 79: 17-22, 2016.

20. Jiang W, Wang Z and Jia Y: CEP55 overexpression predicts poor prognosis in patients with locally advanced esophageal squamous cell carcinoma. Oncol Lett 13: 236-242, 2017.

21. Sun Z, Ji N, Bi M, Zhang Z, Liu X and Wang Z: Negative expression of PTEN identifies high risk for lymphatic-related metastasis in human esophageal squamous cell carcinoma. Oncol Rep 33: 3024-3032, 2015.

22. Akhtar J, Wang Z, Zhang ZP and Bi MM: Lentiviral-mediated RNA interference targeting stathmin 1 gene in human gastric cancer cells inhibits proliferation in vitro and tumor growth in vivo. J Transl Med 11: 212, 2013.

23. Rice TW, Rusch VW, Ishwaran H and Blackstone EH; Worldwide Esophageal Cancer Collaboration: Cancer of the esophagus and esophagogastric junction: Data-driven staging for the seventh edition of the American Joint Committee on Cancer/International Union against cancer cancer staging manuals. Cancer 116: 3763-3773, 2010 .

24. Nieman DR and Peters JH: Treatment strategies for esophageal cancer. Gastroenterol Clin North Am 42: 187-197, 2013.

25. Fesik SW: Promoting apoptosis as a strategy for cancer drug discovery. Nat Rev Cancer 5: 876-885, 2005.

26. Cajone F and Sherbet GV: Stathmin is involved in S100A4-mediated regulation of cell cycle progression. Clin Exp Metastasis 17: 865-871, 1999.

27. Belletti B and Baldassarre G: Stathmin: A protein with many tasks. New biomarker and potential target in cancer. Expert Opin Ther Targets 15: 1249-1266, 2011.

28. Zhang C, Chakravarty D, Sakabe I, Mewani RR, Boudreau HE, Kumar D, Ahmad I and Kasid UN: Role of SCC-S2 in experimental metastasis and modulation of VEGFR-2, MMP-1, and MMP-9 expression. Mol Ther 13: 947-955, 2006.

29. Shaw RJ and Cantley LC: Ras, PI(3)K and mTOR signalling controls tumour cell growth. Nature 441: 424-430, 2006.

30. Janzen V and Scadden DT: Stem cells: Good, bad and reformable. Nature 441: 418-419, 2006.

31. Manning BD and Cantley LC: AKT/PKB signaling: Navigating downstream. Cell 129: 1261-1274, 2007.

32. Di Cristofano A and Pandolfi PP: The multiple roles of PTEN in tumor suppression. Cell 100: 387-390, 2000.

33. Shah A, Swain WA, Richardson D, Edwards J, Stewart DJ Richardson CM, Swinson DE, Patel D, Jones JL and O'Byrne KJ: Phospho-akt expression is associated with a favorable outcome in non-small cell lung cancer. Clin Cancer Res 11: 2930-2936, 2005.

34. Tang JM, He QY, Guo RX and Chang XJ: Phosphorylated Akt overexpression and loss of PTEN expression in non-small cell lung cancer confers poor prognosis. Lung Cancer 51: 181-191, 2006.

35. Panigrahi AR, Pinder SE, Chan SY, Paish EC, Robertson JF and Ellis IO: The role of PTEN and its signalling pathways, including AKT, in breast cancer; an assessment of relationships with other prognostic factors and with outcome. J Pathol 204: 93-100, 2004. 